\title{
Femina et son tournoi des poétesses : simple stratégie médiatique? Le cas exemplaire d'Hélène Picard
}

\author{
Nicole LAVAL-TURPIN \\ Chercheuse indépendante \\ lavalturpin@yahoo.fr
}

\section{Resumen}

Creada el uno de febrero de 1901, la revista Femina quiso promover un nuevo perfil femenino y se preocupó por otorgarle un espacio regular entre sus páginas a la poesía que oscilaría entre una función ornamental y un verdadero campo de creación. Condesa de Noailles, reina de Rumanía, los títulos de sus colaboradoras le aseguraban el prestigio, hasta que un concurso de poemas se abriera (el 15 de enero de 1903) a cada lectora. El éxito de dicho "torneo" animó a nuevas creadoras, entre las cuales encontramos a Hélène Picard, quien obtuvo el Premio Nacional de Poesía en 1907. Su compendio L'Instant éternel le abrió las puertas de los salones parisinos y, después, el mundo de la escritora Colette. En este artículo analizamos cuál fue el papel preciso de la revista Femina (apoyo, límites) en este recorrido que el choque del Surrealismo llevó a su fin.

Palabras claves: concurso poético, promoción, emancipación creadora.

\section{Résumé}

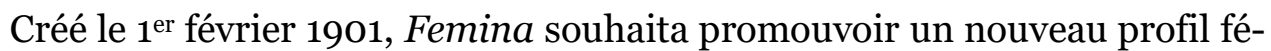
minin. Elle tint à réserver une place régulière à la poésie. Fonction ornementale ? ou champ de création ? Comtesse de Noailles, reine de Roumanie, les noms à particules des contributrices en assuraient le prestige, jusqu'à ce qu'un concours de poèmes fût ouvert (15 janvier 1903), à chaque lectrice. Le succès de ce " tournoi » engendra des créatrices nouvelles. Telle Hélène Picard, qui obtint le prix national de Poésie en 1907 ! Son recueil L'Instant éternel lui ouvrit d'abord les salons parisiens, puis le monde de Colette avec qui elle se lia. Quel fut donc le rôle précis, la caution et les limites de Femina dans un tel parcours qu'éteignit le choc du Surréalisme?

Mots clé : concours poétique, promotion, émancipation créatrice.

\section{Abstract}

Born on 1 February 1901, the purpose of Femina was to promote a new feminine type so, they prioritized Poetry. Was it just for embellishment? An open space

\footnotetext{
*Artículo recibido el 17/06/2021, aceptado el 19/11/2021.
} 
for creation? Comtesse de Noailles, Queen of Romania, double-barrelled names, worked towards its prestige until a poetry contest on 15 January 1903. Any female reader could enter the competition. It was a success and some new poetesses came to light: Hélène Picard got the National Poetry Award in 1907. Thanks to her book L'instant éternel, she could enter famous «salons parisiens» and approach the writer Colette. The point: what were the exact function, support and limits of Femina before the advent of Surrealism?

Keywords: poetry contest, promotion, creative emancipation.

Il ne vous atteint pas, l'affreux cri des sirènes Dans les bars de cristal, éclatants perroquets, Frivoles favoris des sombres capitaines.

Hélène Picard, Délivrance.

Voilà qu'elle est morte, celle qui semait, avec un grand air de faste et d'indifférence, des vers comme ceux-là. [...] Il m'est incompréhensible qu'elle soit morte, et je serai longue à le comprendre. [...] Une vie aussi pure que la sienne ne peut manquer de paraître mystérieuse. [...] Autour d'elle, les traces de la poétique abondance voltigeaient. Une de ses matinées suffisait, souvent, à ensemencer la chambre bleue. Chez cette fille de la solaire Ariège, qui se couchait tôt, s'éveillait avec les perruches, la lumière du matin mûrissait un poème. [...] C'est cette Hélène-là qui vivait secrète sous le gorgerin pailleté de $M^{m e}$ Hélène Picard, lauréate en 1904 d'un jury de femmes, tant lettrées que mondaines. Femina la hissa aux côtés des baronne de Baye et des Daniel Lesueur, pas bien loin d'un dîner photographié chez $\mathrm{M}^{\mathrm{me}}$ de Pierrebourg, alias Claude Ferval.

Ainsi s'émeut Colette (1988 [1958] :11-26) dans un hommage à son amie Hélène, qu'elle avait reçue pour la première fois fin 1919, dans les bureaux du Matin, quotidien où elle tenait alors la rubrique littéraire "Contes des mille et un matins ». Bien qu'elle se considérât toujours - ou le feignît comme « une sorte de monstruosité » qui n'avait «jamais écrit de vers »1, c'est pourtant grâce à leur amitié et leurs profondes affinités littéraires que la poétesse Hélène Picard (1873-1945), révélée par la revue Femina, n’a pas totalement sombré dans l'oubli, une fois éteints les feux d'une Belle Époque propice aux femmes poètes ${ }^{2}$.

1 «La poésie que j'aime », conférence donnée le 10 décembre 1937 à l’Université des Annales.

${ }^{2}$ Le milieu universitaire et les Cahiers Colette de la Société des amis de Colette poursuivent l'étude de ces liens que la prosatrice entretint avec ses amis et le monde littéraire qu'elle fréquentait. 


\section{Femina et les mécanismes d'un prestige \\ 1.1. Le sceau de l'élégance}

Colette tout comme Hélène recevait Femina dans des tubes en carton fraîchement encollés, aussi lut-elle bien avant leur rencontre ses vers mis à l'honneur par la revue. Les pages réservées aux poétesses faisaient la part belle à ces " ouvrages de dames » signés de noms à particule, d'Anna de Noailles à la reine de Roumanie. Ils conféraient au périodique un certain cachet, propre à satisfaire un lectorat bourgeois, se plaisant à découvrir les villégiatures célèbres, les salons en vue et les talents prometteurs - tout cela dans l'assurance du bon-goût. Les poèmes insérés confirment le parti-pris éditorial : leur « classicisme » dans le style des muses plaintives du Romantisme relève de l'ornement ; les commentaires attenants ne sont qu'éloges convenus, dont l'excès prête aujourd'hui à sourire. Ainsi dans la livraison du 15 juin 1904 découvre-t-on un « exquis » ménage de poètes, où « $\mathrm{M}^{\mathrm{me}} \mathrm{Ed}-$ mond Rostand exquisément poète s'est faite la Muse exquise de son mari »! Ces hyperboles figeaient d'emblée la poésie dans un cadre étroit d'apparente excellence, inaccessible à de plus modestes ambitions.

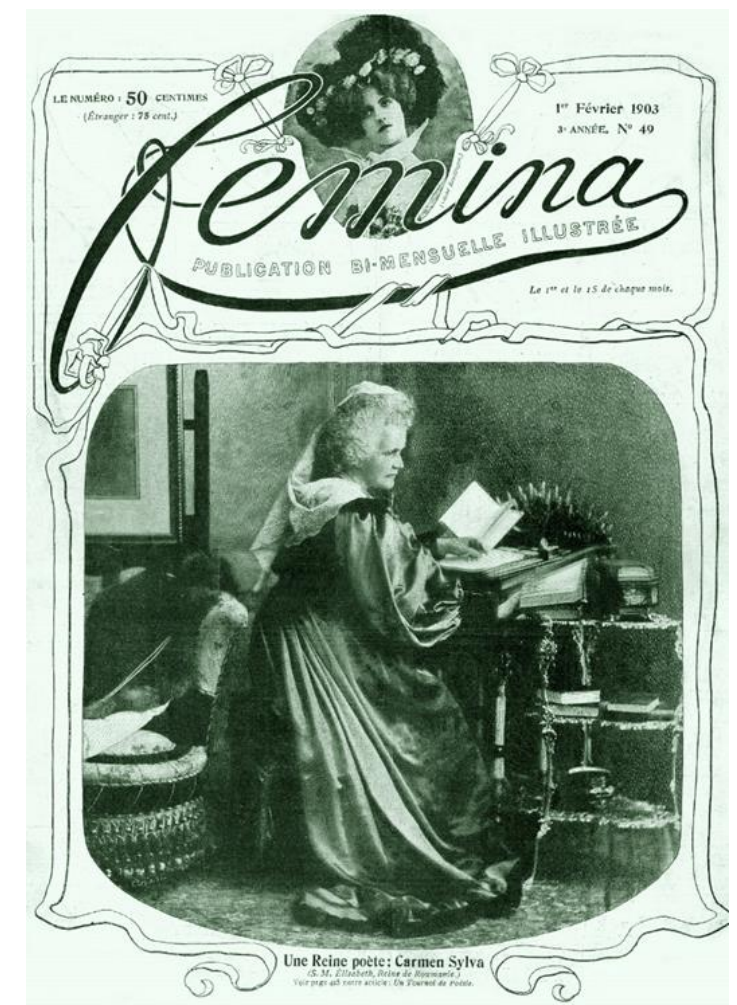

Image 1 : photo de Carmen de Roumanie.

Voici une photo de couverture $\left(\mathrm{n}^{\circ} 49,1^{\text {er février } 1903) \text { tout à }}\right.$ fait représentative de la tendance en usage : la reine Élisabeth de Roumanie, dotée de son modeste nom de plume, apparaît dans les emblèmes de son noble loisir : pupitre, livre ouvert, cahier sous la main ; le visage concentré révèle la méditation, et les atours chatoyants disent la royale origine. Femina cultive toujours l'élégance de l'image.

Éloquente, la légende valorise une singularité : être « Reine poète » tient de l'oxymore et de la grande classe. Son titre officiel, précisé ensuite, $\mathrm{S}$. M. Élisabeth, Reine de Roumanie, ne figure qu'au second plan, mais grandit le portrait: la modestie sied à la vraie noblesse.

\subsection{Une initiative bienvenue}

Or il se trouva qu'une rubrique nouvelle bouscula cette image vieillotte, créant un phénomène dont la popularité fut stupéfiante. Une simple action promotionnelle - l'idée d'un concours poétique ouvert à toutes les lectrices - eut un tel retentissement que l'expérience, annoncée le 15 janvier 
1903, devint une institution annuelle. Une lauréate s'y fit un nom qui l'introduisit dans le monde des lettres : Hélène Picard. Son parcours de provinciale peut servir d'emblème à cette stratégie médiatique qui, au-delà, servit de tremplin socio-culturel à une génération nouvelle de femmes en quête de légitimité.

Baptisée tournoi, l'entreprise n'avait rien de bouleversant. Sous l'égide obligée de noms choisis - comtesse Anna de Noailles, baronne de Baye, $\mathrm{M}^{\mathrm{me}}$ Rostand et $\mathrm{M}^{\text {lle }}$ Gautier, entre autres - et selon quatre domaines distincts, il s'agissait de composer un sonnet, un monologue versifié (ou une saynète), des vers à chanter ou un ensemble de vers à sujet imposé. Formes traditionnelles et jury de luxe ne risquaient guère d'engendrer la moindre

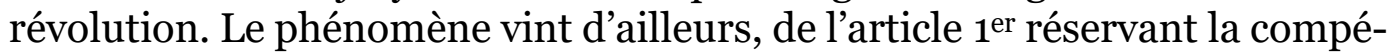
tition aux seules femmes, sans distinction de nationalité, et jugé par des femmes. Outre la promesse de nombreux prix, brillait la perspective d'une insigne promotion : la lauréate siègerait l'année suivante au jury. Les hommes aux commandes des prix littéraires, et majoritairement détenteurs du genre poétique, disparaîtraient le temps d'un tournoi...

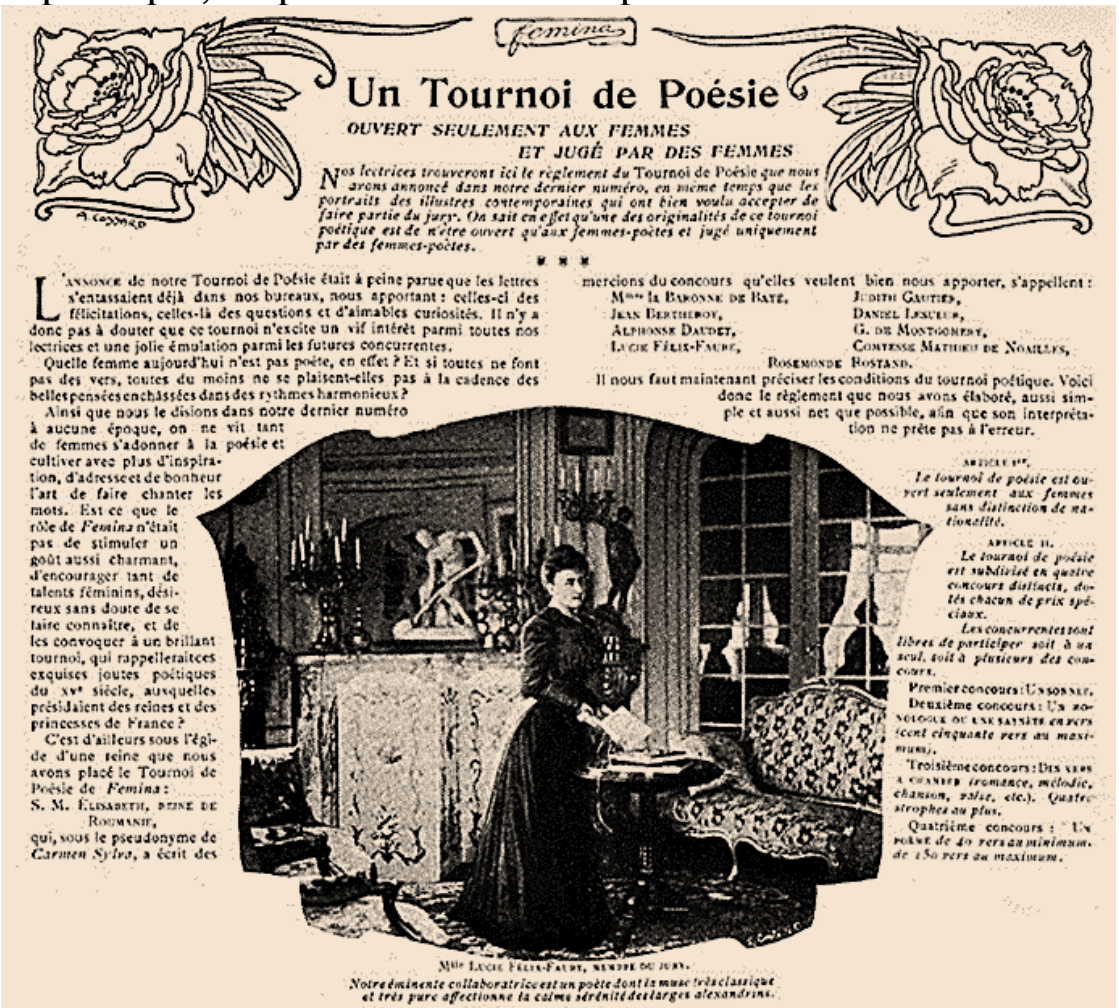

Une demi-page annonçait l'événement, selon les bienséantes conventions : un titre sous frise florale à la mode, et une large place accordée à la photographie d'une des poétesses au jury. Sa pose est classique, livre à la main près d'une table, dans un salon cossu et ordonné. La légende met en valeur sa compétence, et sa qualité d'écriture. On retrouve les hyperboles gages de sérieux : "éminente, très classique et très pure ». 
Dès la quinzaine suivante, les lettres de lectrices affluaient, enthousiastes ou curieuses de plus amples détails. Dès le $1^{\text {er }}$ juin le tirage de Femina montait à cent mille exemplaires. Le palmarès (15 septembre 1903) revint à une certaine Juliette David, parmi les 8 ooo envois recensés, certains venus de Grèce, d'Écosse, d'Algérie. Parmi les pièces primées, figuraient en bonne place dans trois catégories celles d'une Ardéchoise, Hélène Picard. Le numéro ne consacre pas moins de 7 pages aux divers résultats scrupuleusement présentés : nombre d'envois, de récompenses, de mentions, avec les noms des femmes primées et les vers de la lauréate. L'écrivain Henri Barbusse tient alors le bloc-notes littéraire de la revue : désigné secrétaire du jury, il confirme un tel succès : l'initiative s'apprêtait à devenir institution chaque année reconduite, sur des critères simplifiés - une seule composition de 150 vers. Dans un même souci de valoriser le monde féminin, le thème consisterait l'année suivante en un hommage à George Sand, dont la naissance en 1804, soit cent ans plus tôt, ne manquerait pas d'être célébrée. Et le 15 juillet 1904 précisément, Hélène Picard remportait la palme.

\subsection{Les retombées du tournoi}

À lire de près les articles liés à cette initiative, et au regard des poèmes primés, régulièrement cités dans les numéros suivants jusqu'au concours de 1904, on mesure les limites de l'opération, sans en nier l'importance. Soucieuse d'encourager et valoriser la vague imprévue de concurrentes ayant répondu à l'appel, Femina multiplia les distinctions, ici dans le numéro du 15 septembre 1903 :

Nous n'avons pas hésité à récompenser un grand nombre de concurrents et nous avons été ainsi amenés à accorder pour chaque section des prix supplémentaires ainsi que des mentions à tous les envois que le jury nous a désignés. Le nombre de ces prix supplémentaires s'élève à 26. Le nombre total des mentions est de 823 . La place nous manque ici pour donner de nouveau la liste des beaux ouvrages attribués à ce concours. [...] En outre nous avons créé un Diplôme Femina en couleurs, dont la composition a été confiée à un de nos plus brillants artistes : le dessinateur Orazzi.

On peut sourire de ces accessits bienveillants soulignant surtout la générosité du journal (qui maintenait ainsi sa stratégie de vente !) ; y lire aussi un remerciement implicite à toutes ses lectrices enthousiastes, dont les gratifiants courriers perdurèrent après le palmarès. Mais il faut relativiser nettement la valeur poétique des envois. Aucun ne s'écarta des thèmes rabâchés depuis le romantisme et repris par Francis Jammes, appels du cœur, vibrantes bucoliques, extases ferventes. Juliette David, la grande gagnante, inscrit un distique de Vigny en exergue de ses 30 quintils. Les poétesses en herbe imitèrent sans originalité le style qu'elles croyaient propres 
à la poésie, classique et bienséant. Illisibles aujourd'hui, leurs vers justifiaient déjà l'ironique sanction masculine, évoquant ces «ouvrages de dame », simple broderie même sous l'autorité de « La Maison du Berger ».

Toutefois dans son compte rendu poursuivi le mois suivant (livraison du 15 octobre 1903) Barbusse ébauche un point nouveau : les concurrentes ont révélé un lectorat très varié, qui ne se cantonnait pas à une bourgeoisie de haut vol. Elles ne prétendent pas à une carrière de femmes de lettres. " Petite ouvrière » ou " domestique ", l'écriture leur aura simplement ouvert, le temps d'un tournoi, un espace de liberté, un rêve accessible. Loin d'un projet plus créatif - ou d'une velléité d'ascension - elles prennent soin, précise Barbusse amusé, d'affirmer qu'elles continueront leur métier. Sage promesse de rentrer dans le rang, navrant réflexe à nos yeux de femmes émancipées, cent ans après ! La revue ne cultivait pas le moindre féminisme militant, suffragettes et « amazones » n'en faisaient pas la une. Mais l'allusion à ces courriers naïfs n'est pas forcément négative : elle témoigne, même à très bas bruit, d'un nouveau milieu féminin amené peu à peu, même sans nom à particules ou notoire accointance, à trouver sa voix poétique. Le déferlement d'autrices que Paul Flat en 1909 voit comme « un fait social » prend son infime germe, dans ce type d'expériences.

Enfin, Barbusse, indéfectible complice des femmes dans l'euphorie de ce premier tournoi, assaisonne quelques lecteurs mâles - et bien sûr anonymes - incriminant le choix du jury, et jouant à tort les censeurs à propos de métrique et d'orthographe. Il se réjouit même d'un désabonnement annoncé : «Allons tant mieux, cher Monsieur X, tant mieux! » Encore un signe, bien que modeste, vers le changement ?

\section{Hélène Picard et Femina : une histoire singulière}

Quand la revue, dans son numéro du 15 juillet, proclame Hélène Picard lauréate pour 1904, le succès prend immédiatement effet : ses 28 quatrains sont mis à l'honneur aux fêtes du centenaire de George Sand, déclamés par une comédienne en vue, $\mathrm{M}^{\mathrm{me}}$ Baretta-Worms.

La page consacrée à l'annonce met cette fois l'accent sur les vers couronnés, non sur le portrait - en simple médaillon - de l'autrice. Il ne s'agit plus en effet d'un membre prestigieux du jury, mais d'une provinciale jusque-là inconnue ! La hiérarchie sociale se glisse dans les détails.

Le thème du tournoi, imposé cette année-là, susceptible d'intimider des plumes peu familières de la dame de Nohant, n'avait inspiré que 437 créations, le score final se jouant à un point entre les quatre meilleurs manuscrits. Le jury distribua tout de même un total de 58 mentions ; la revue maintint aussi les enthousiasmes en ajoutant à ses pages, dès le $1^{\text {er }}$ octobre, une chronique nouvelle. Acceptons l'emphase de l'annonce, bien dans le style du temps :

Nous assistons ainsi en ce moment [...] à l'un des plus considérables mouvements en faveur de la poésie que 
l'on puisse signaler dans l'histoire de la littérature. En ouvrant aujourd'hui une rubrique "Poésie » qui sera suivie régulièrement, Femina n'avait donc mieux à faire que de demander à quelques-unes des plus connues parmi les femmes poètes de notre époque de lui envoyer quelques vers.

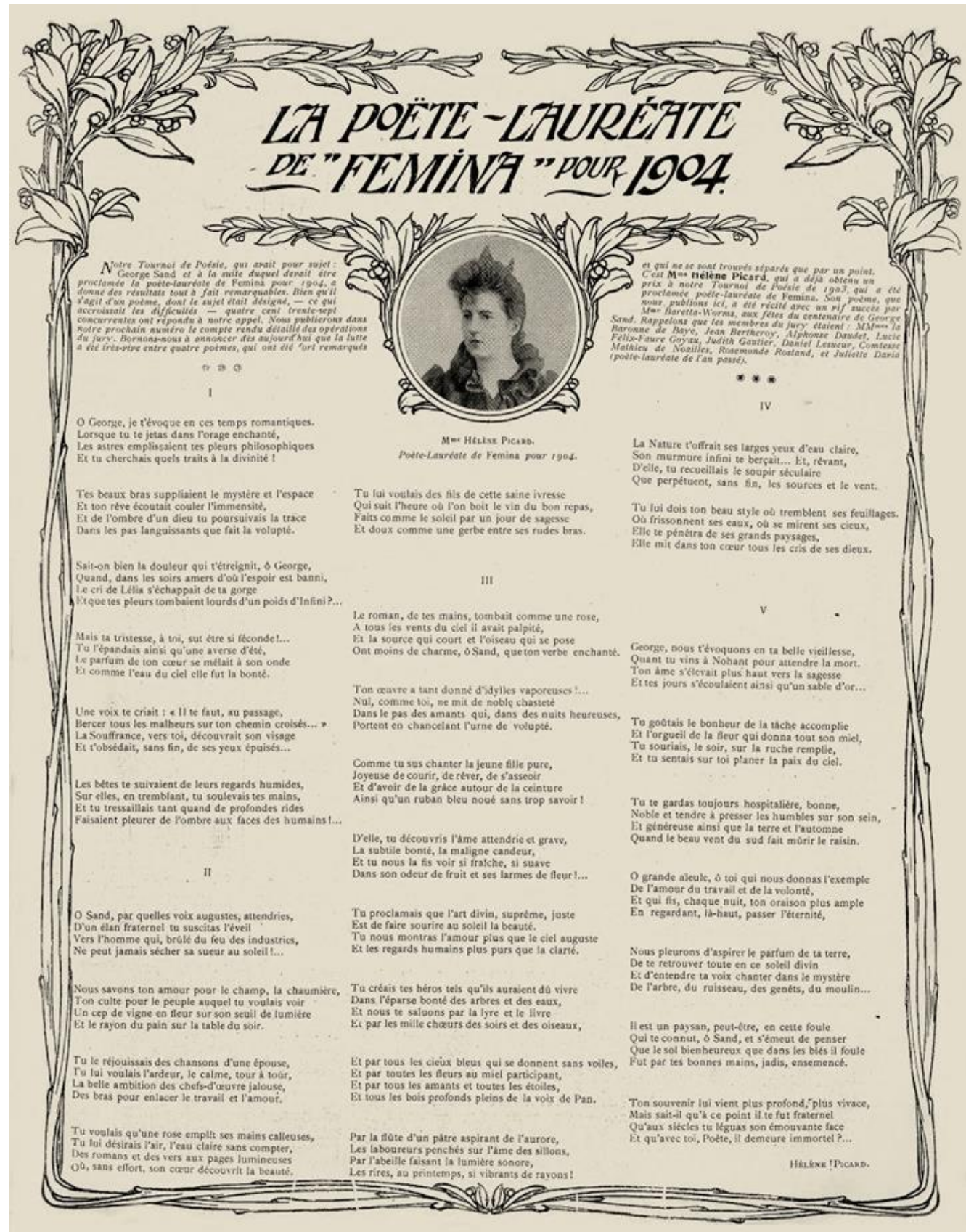

Ainsi le genre poétique, de flagrante manière, constituait surtout le fleuron de la revue, restait caution de son prestige, bref, jouait toujours les 
ornements. Le destin de la nouvelle élue pouvait-elle échapper à cette couleur bien trop générale?

\subsection{Un parcours préparé}

Hélène Picard avait 31 ans lorsqu'elle gagna le tournoi. Loin de l'avoir envisagé comme un simple passe-temps de lectrice, elle y vit plutôt une belle opportunité d'ajouter une expérience à son art : elle s'adonne en effet à sa vocation poétique depuis plusieurs années. Entre 1896 et 1898, cette méridionale fréquente à Toulouse L'Effort, cercle et revue de poètes contre l'art pour l'art, cultivant l'énergie et l'amour de la vie pour l'individu et la société. Elle a rencontré là son époux Jean Picard, lui-même poète et membre du groupe. Primée deux fois à l'Académie des Jeux Floraux (un fleuron régional) en 1899 et 1900, elle parachève une pièce lyrique en cinq actes et en vers, La Feuille morte, qui paraîtra en 1903 grâce à un typographe de Privas où elle a suivi son mari promu conseiller de préfecture en Ardèche. L'œuvre à portée confidentielle obtient toutefois l'approbation d'un Jules Lemaittre (selon les auteurs d'une Anthologie des poètes du Midi) et même d'Émile Faguet dans Le Journal des débats (11 septembre 1903) : " Pour ce qui est du don, il est là » conclut-il. Dès lors Hélène ne cesse plus d'écrire, adonnée simultanément à deux recueils dont l'un consacrerait bientôt son talent.

En attendant, elle se doit à Femina. Le tournoi 1905 est annoncé dans le numéro du $1^{\mathrm{er}}$ mai, sur une page agrémentée de son seyant portrait.

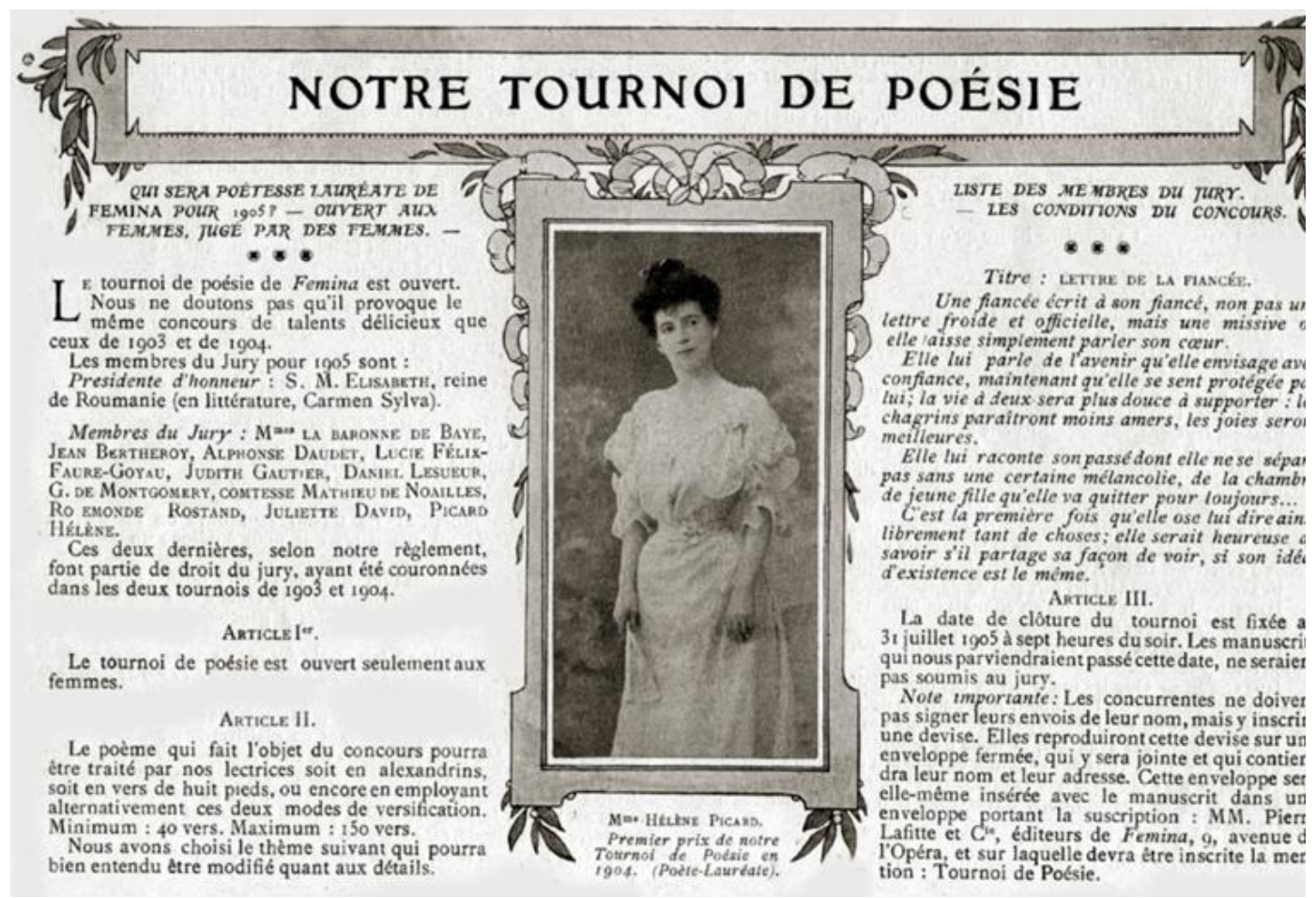

Hélène Picard, maintenant reconnue et intégrée au jury, bénéficie donc d'un cliché plus valorisant que le premier. En robe d'apparat, pochette 
à la main droite, tête inclinée avec grâce, elle se prête aux codes du portrait traditionnel. Pris en studio : la poétesse n'a pas le privilège d'être vue dans l'intimité de son intérieur bourgeois.

Désormais membre du jury, Hélène Picard peut ainsi siéger près d'Anna de Noailles avec laquelle elle entretiendra une manière d'amitié, surtout lorsque Colette les rapprochera encore, dans les années 20. Cette première introduction en milieu parisien ne dut guère l'intimider : d'une nature sociable et spontanée, rompue par ailleurs aux exercices mondains incombant à une épouse de conseiller de préfecture, elle ne souffrit certainement pas de son statut de provinciale, selon les condescendants critères attachés à ce vocable. Et elle pouvait percevoir, même de loin, que la Parisienne gagnait peu à peu les devants de la scène sociale, avec l'apparition, selon Julie Bertrand-Sabiani (1998 : 264) des «penseuses, amazones, journalistes et bas-bleus envahiss[ant] le champ littéraire ».

\subsection{6, ou les avatars d'un manuscrit}

Hélène Picard ne cesse jamais d'écrire. Dès le printemps 1906, elle tient prêt un manuscrit intitulé L'Instant éternel et le propose à un nouveau concours : tous les ans, une commission chargée par le ministre de l'Instruction publique d'attribuer la Bourse nationale du voyage littéraire se réunit à la Société des gens de lettres afin d'élire son lauréat. Le prix s'élève à trois mille francs et doit, en vertu du règlement, revenir cette année-là à un poète. Jules Bois rapporte dans le Gil Blas du 2 juillet un point décisif de la séance. On étudie le recueil d'Hélène parvenu, prend-il soin de préciser, « au dernier moment » :

Lorsque Émile Blémont ${ }^{3}$ [...] nous confia à chacun, par ordre alphabétique, une série de recueils à examiner, Émile Michelet trouva, dans son lot, L'Instant éternel de $\mathrm{M}^{\mathrm{me}}$ Hélène Picard. Il fut ravi et n'eut qu'à nous communiquer quelques extraits pour nous faire partager son ravissement. Déjà la bourse était virtuellement donnée.

Or le jury préfère que le mérite de l'œuvre reste une affaire de femmes! Jules Bois poursuit en effet de la sorte, assez peu élégante : « $F e$ mina et La Vie heureuse ne se préparent-elles pas à couronner de billets de mille le front d'un poète ? $\mathrm{M}^{\text {me }}$ Hélène Picard est toute indiquée ». On semble ignorer en ce lieu que Femina, selon ses statuts, ne peut offrir de seconde chance à une candidate déjà primée. Quant au prix "Vie heureuse », il est bien décerné à une poétesse, mais il s'agit d'André Cortis.

La même année, le manuscrit de L'Instant éternel concourt pour le prix national de la poésie - prix de l'Académie française dit « Archon-Despérouses »- qu'Anna de Noailles avait reçu en 1902 pour Le Coeur innombrable. L'ouvrage d'Hélène parvient en sélection finale mais une fois encore, un homme est gagnant, Abel Bonnard, pour Les Familiers, curieux bestiaire

3 Poète ami de Verlaine, président de la commission. 
aux échos symbolistes. Manquer de si peu un prix à deux reprises signe cependant l'intérêt porté à l'ouvrage. Il aura sa revanche l'année suivante.

\subsection{7, l'année charnière}

L'Instant éternel paraît sous sa forme de recueil en février 1907 aux éditions Sansot et Cie. Le fameux prix Archon-Despérouses, doté de quinze cents francs, lui échoit enfin, quelques mois plus tard, devant quatre-vingtdeux autres ouvrages de vers, " grâce à la spontanéité et l'abondance de son lyrisme ». Ainsi parle Maurice Barrès lui-même, en séance publique du jeudi 21 novembre de l'Académie française. Il n'est pas certain qu'il s'exprimât en son nom propre, lui qui s'agaçait de ce flux de poétesses faisant de l'ombre à son égérie : « Toutes ces servantes méconnaissent donc leur reine qu'elles ne gardent pas le silence ! ", écrit-il à Anna de Noailles le 23 juin 1906. En tout cas, ce couronnement coïncide avec une floraison poétique particulièrement féconde en cette « année tournante ». Ainsi Clément Delmas (1987 : 684) nomme-t-il l'année 1907 ,

[...] une étape importante non seulement dans la littérature féminine, en particulier la poésie. Même si l'usage de [leurs] droits rencontre encore des résistances tenaces [...] à travers tout ce qu'on écrit, aspirations et critiques, un type féminin se dessine, un idéal accessible à toute femme, une sorte de «femme moyenne », comme on dit « un Français moyen » : c'est la femme consciente.

La figure de la nouvelle lauréate répond parfaitement à ce profil. $\mathrm{Fe}$ mina n'offre quasiment plus de numéro sans poème de femmes, aux noms certes bien oubliés aujourd'hui. Mais des œuvres plus saillantes ne sont pas en reste : dans sa livraison du 15 juin, la revue signale sur une page et demie, avec un portrait des poétesses, et par la plume élogieuse de l'écrivain Henri Duvernois, la sortie de L'Instant éternel, en citant quelques strophes. Elles jouxtent celles d'Anna de Noailles tirés des Éblouissements, parus la même année. En outre le 11 juin s'était tenue au théâtre Femina une matinée en l'honneur de Sarah Bernhardt : Madeleine Roch de la Comédie-Française y avait déclamé un poème commandé pour l'occasion à Hélène. La fidélité du journal qui l'avait lancée ne se démentit pas par la suite, même si elle fut discrète : multipliant les occasions de valoriser les gagnantes de son tournoi, Femina avait institué en 1908 une fête annuelle de la poésie, avec lectures de poèmes primés. L'expérience, renouvelée le 2 février 1909, remit à l'honneur Hélène Picard, devenue un emblème de réussite ! Hélène Avryl, dans son compte rendu du $1^{\mathrm{er}}$ mars, encense les vertus du tournoi en ces termes : « N'est-ce pas par l'un d'eux que fut mis en lumière, pour la première fois, le talent violent et touchant, audacieux et complexe, de Mme Hélène Picard, dont la renommée est désormais considérable ? » Oublions l'hyperbole, pour ne retenir qu'un phénomène d'inversion : le nom d'Hélène redevable 
au début à un savoir-faire médiatique lui servait à présent de fleuron promotionnel.

\section{L'Instant éternel : miroir d'une époque ou titre de choix ?}

L'oubli où l'œuvre a sombré offre la réponse : de la Belle Époque et de son flux de poétesses, seule demeure Anna de Noailles. Un chef d'œuvre aurait survécu. Mais le regard moderne fausse la lecture, un centenaire et quelques révolutions poétiques plus tard. La critique doit dépasser aussi le fait d'une ascension dérisoire, et la question logique : un simple concours organisé pour un public ciblé, par une revue bien-pensante, dans un but somme toute commercial, avait certes mis un nom en lumière, mais garantissait-il une insigne vocation?

\subsection{Gros plan sur L'Instant éternel}

L'empreinte romantique sous l'égide de Musset saute aux yeux et irrigue discrètement maints poèmes. Titre conjuratoire, L'Instant éternel retrace minutieusement les étapes d'un amour, de son efflorescence à sa retombée, en six sections, au rythme de l'alexandrin, suite d'extases ou litanies. Rien de bien novateur ! Pourtant une ligne plus originale émerge des images érotisées, de leur sensualité prégnante rappelant les ardents échos d'une Louise Labé : le corps glorieux de l'amant en sa force animale est chanté en termes nets, répondant à la jouissance féminine, fraîchement révélée :

Ô vous le dévêtu, vous le nu, je vous vois, [...]

Ô jeune corps de joie où la splendeur circule

Je te glorifierai dans la vague du blé [...]

Ô jeune fleur de vie, ô chair pure et sacrée.

La volupté se dit dans la courbe éloquente d'un décasyllabe :

Le désir qui croît, le vouloir qui sombre

Entre des bras nus.

Autant de vers - sans compter certains titres, «La Bonne Joie », «Pénétration », «Le Trouble »- qui n'avaient aucune chance de paraitre dans Femina... même si l'amant fait dieu semble droit venu du Cantique des Cantiques :

Votre bouche m'aura pour toujours abreuvée

Je vous ai fait ma couche et ma table servie,

Vous fûtes ma maison et je vous ai planté,

À jamais, comme un arbre au milieu de ma vie.

La poétesse marque là son appartenance à une génération de femmes qui osaient enfin évoquer la vie de leurs sens, magnifier l'autoérotisme ou les blasons du corps masculin. Fut-elle donc plus expressive qu'une autre, dans l'impudeur du désir ? Il semble que non : Marie Dauguet, sous le titre éloquent Par l'Amour, avait cultivé dès 1904, la même ferveur biblique, tel son « Cantique au bien-aimé de la reine de Saba » : 
Bienheureuse la bouche où s'attache ta bouche

Qui goûte tes baisers semblables à l'encens,

Au cinname, à l'odeur des pêches mûrissant.

Hélène devait aussi connaître Le Cour innombrable ayant valu à Anna de Noailles le prix Archon-Despérouses en 1902, ce qui l'avait peutêtre incitée à y prétendre aussi. "L'Offrande à la nature » fait en tous cas un pur écho à sa propre voix :

J'ai connu les désirs qui brûlent dans vos soirs

Et qui font naître au cœur des hommes et des bêtes

La belle impatience et le divin vouloir.

Et sous forme de versets, Marguerite Burnat-Provins, dans Le Livre pour toi (paru comme L'Instant éternel en 1907) attise les mêmes feux :

J'ai regardé tes lèvres qui plient sous les miennes, tes dents où mes dents se sont heurtées illuminent ton sourire, ta langue chaude m'endort, et quand je m'éveille de mon vertige, c'est pour revoir ton corps triomphant, altier comme un pilier d'ivoire...

Ultime parallèle, sans clore la recension possible, Hélène PerdrielVaissière la même année chantait Celles qui attendent par le don de son jeune corps, au cœur de « L'Été » :

Et j'ai gardé la clé du jardin qui t'attend, Je t'offre les frissons de la saison heureuse, Cueille-la tout entière en une seule fleur.

Certes ces strophes peuvent paraître peu hardies, voire maladroites dans leur sensible souci d'audace. Cette faiblesse, Jean Dornis, pseudonyme de $M^{\text {me }}$ Guillaume Beer (1912 : 190-191), femme de lettres elle-même, l'attribuera plus tard non sans lucidité à un complexe de l'état féminin :

Cette inconscience de la valeur empêche la femme nouvelle d'épanouir ses forces [...]. La femme ne sait inventer, dit l'homme, elle ne peut pas avoir du génie. Alors [...] elle sent que pour attirer l'attention, il faut qu'elle étonne, qu'elle pousse des cris si hauts, si étrangers, qu'on ne puisse pas ne pas l'entendre, ne pas lui accorder ce crédit qui obligera le public à lire une œuvre, une originalité dont elle-même, sans cesse la femme doute.

L'abondance de commentaires, quand parut L'Instant éternel, offre un juste reflet des jugements à ce propos.

\subsection{La réception du recueil}

Dans la mesure où l'œuvre ne s'avérait pas si distincte de la foisonnante moisson poétique de 1907, comment interpréter son succès critique? Elle devint sans doute le creuset de cette écriture féminine émergente, concentrant les enthousiasmes, sorte de synthèse équilibrée des autres parutions. Elle entraîna aussi un phénomène identitaire. Il arrive que certains 
titres soient « dans l'air », souligne Fernand Gregh chroniqueur des Lettres, le 15 avril 1907 ; ils émergent à point nommé. Trop souvent l'élégie plus que l'hymne présidait jusque-là à l'expression amoureuse. L'ouvrage d'Hélène respirait une fraîche authenticité propre à rendre heureux. Émile Faguet consacra d'ailleurs quinze pages de La Revue latine (25 février 1908) à cette " paysanne latine [...] aimant de source ». Elle préservait surtout - un réel avantage selon les masculins commentaires ! - de véhémences plus marquées, féministes avant le nom, chez René Vivien ou Lucie DelarueMardrus. Son sage tumulte devenait même providentiel, en se démarquant des partis pris d'Amazones que Jules Bois suggère en trait d'humour (livraison du Gil Blas, o2 juillet 1906) : " Une poétesse extraordinaire nous est née. Elle chante l'Homme, elle ne le débine pas. Mon dieu ! Merci... !».

Ainsi la presse - quotidienne et périodique, versée ou non en poésie - salua une année durant, avec bienveillance, la nouvelle venue des Lettres. Femina, on l'a vu, avait tôt mentionné le succès de son " poulain »; le même mois (juin 1907) le mensuel La Vie heureuse, pour le même type de lectrices, confiait à Séverine l'éloge d'une " gloire neuve ». Mais cette fois les échos excédèrent le cadre d'un journal féminin. Des articles signés de noms établis fleurirent entre autres dans L'Intransigeant (Albert Flament, 29 mars 1907, avouant ne pas connaître d'homme « lisant ces vers qui ne désirera d'être aimé comme cette femme-poète aime »), La Dépêche $d u$ Midi, (Victor Margueritte, 26 mai 1907). Dans Le Gaulois du 25 avril, Gérard d'Houville (Marie de Heredia) est la seule à évoquer à juste titre le masochisme inhérent à la passion : "Je ne suis que trop persuadée que les femmes les plus charmantes ont une préférence secrète pour les hommes qui les font souffrir. Dans Le Journal, Catulle Mendès lui prête « une fluidité qui fait songer aux abandons négligés de la tendre et grande Marceline ». Fernand Gregh dans sa chronique des Lettres du 15 avril accorde à Hélène Picard la même grâce " d'être femme dans son œuvre ". Poésie signale dans sa livraison d'avrilmai-juin L'Instant éternel comme un gros livre de tendresse. L'auteur TounyLeyris prédit qu'aux « soirs d'adieu », on se souviendra de ses vers. Je sais tout dirigé par Henri Barbusse agrémente sa rubrique mondaine « Lettres et Arts » d'un portrait de la poétesse (15 mars et 15 mai 1908). Désormais reconnue «femme de lettres », elle peut apparaitre tenant un recueil ouvert, emblème de son art. La pose reste convenue, le visage empreint

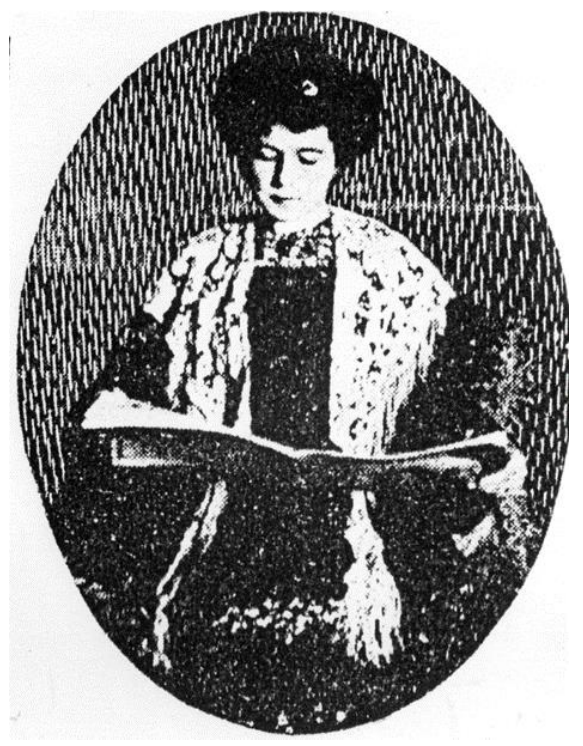
de sérieux concentré sur la page. Le format ovale du cliché achève de prêter 
féminité et charme à l'ensemble.

Les impressions convergent : ferveur communicative d'une passion aiguë, vigueur émouvante dans l'abandon de soi, fluide grâce de l'écriture... Un fantôme, la grande aînée des années 30 Marceline Desbordes-Valmore, revient sous la louange comme une réminiscence qui marque le vivace engouement dont jouissait encore la veine romantique. Mais comment y lire aujourd'hui un signe très élogieux ? En effet, si la poésie féminine s'identifiait à une mouvance reconnue, elle devenait moins susceptible de déranger, en ces accents parfois hardis ! Autre critère à première vue positif mais, à la réflexion, ambigu car il ne saurait définir un talent : la qualité de provinciale de notre poétesse, fréquemment soulignée! Son origine méridionale lui vaut d'être perçue comme un être authentique, ses racines lui garantissant une sincère inspiration. La brèche modestement ouverte par Femina grâce à ses tournois n'a pas effacé le vieux clivage entre « ce qui se fait » à Paris et ce qui ne peut exister en dehors. En fait, si son statut de non-parisienne attire à Hélène les sympathies critiques, c'est qu'il atteste la pureté de ses intentions, voire son absence d'ambition ! M. Coinsot, dans son article « Poétesses » des Pages modernes en janvier 1908, s'exprime en ce sens :

Qu'elle reste donc là-bas, toujours, afin de nous prouver que la province n'a pas besoin de venir boire l'air de $\mathrm{Pa}$ ris pour prendre du souffle, ni frôler les princes célèbres de la littérature pour se tailler de l'estime parmi l'élite.

L'air de Paris a beau ne pas manquer au talent, l'appartenance à la province ne constitue pas non plus un critère décisif ! De fait - cruel malentendu - L'Instant éternel devient l'enjeu d'un léger règlement de comptes, à l'encontre des snobs et de son intelligentsia. Albert Flament (L'Intransigeant, 29 mars 1907) s'ingénia à décliner les handicaps de la provinciale afin de mieux tailler le compliment :

Je sais qu'elle habite Privas, que son mari est dans l'administration... C'est à peu près tout. [...] Il suffit de moins parfois pour empêcher de couper une page d'un volume. [...] Elle ne fait partie ni de l'aristocratie, ni de la haute banque, ne reçoit pas à dîner, et ne dîne pas en ville.

Et l'œuvre est présentée de semblable façon par Auguste Dorchain le 12 mai 1907 dans Les Annales politiques et littéraires :

L'amour y est trop tendre, trop passionné, trop douloureux et trop noble pour séduire, dès demain, les dispensateurs et dispensatrices ordinaires de la renommée ; j'entends par là, non les critiques, mais de bien plus puissants qu'eux : les gens du monde professionnels et les belles dames littéraires des thés de cinq heures.

Ces belles égratignées ne désigneraient-elles pas, entre autres, le fameux tournoi et son jury huppé ? Dans le même esprit, le Mercure de 
France du 16 mars 1908 mentionne une revue donnée aux Bouffes-Parisiens : Mistinguett y chante sans charité les occupations de ces «néo-précieuses » :

Toutes les connaissances

De maman ce jour-là

Vous font des conférences :

On s'croit à Femina.

On y cause, on y fume ;

Chaqu'mardi, voyez-vous,

Toutes les femm's de plume

Taill'nt des bavett's chez nous [...]

On lit des vers

C'est beau mais cher,

Pour moi je n'sais

Jamais d'qui c'est,

Il y en a tant!

C'que j'sais pourtant,

C'est qu'lorsqu'on baille,

C'est du Noailles.

Tout's ces dam's-là

Écriv'nt des tas

D'chos's qu'on n'lit pas.

Elles font dans Femina, Femina,

Des romans féminins, féminins,

Quel talent

Mes enfants!

Ces goguenardes piques inhérentes au music-hall restaient de bon ton. Plus sérieuse était la réserve souvent énoncée d'une fâcheuse abondance du verbe, que les poétesses, Hélène Picard moins qu'une autre, ne savaient restreindre. Alphonse Séché dans son anthologie déplore sa « verbosité ». Une certaine critique eut aussi la dent dure, accusant le bienveillant Émile Faguet d'avoir manqué de discernement. Le chroniqueur à ParisThéâtre Gilbert Gréville (pseudonyme d'Alice Marie Céleste Durant) sous le titre « La Sottise éternelle » conclut ainsi :

[...] L'Instant éternel ne vaut pas un centime.

Quant à vous cher Émile, apprenez qu'il ne faut

Se montrer complaisant : c'est un vilain défaut.

Cette faiblesse avérée n'empêcha pas L'Instant éternel de figurer dans les anthologies de référence, signées Jean de Bonnefon, Jules Bertaut, Jean de Gourmont. Lucien Maury aura ici le dernier mot ; ainsi privilégie-til «La Gloire de nos poétesses », y incluant Hélène Picard nommément, dans La Revue bleue du 28 mars 1908 :

Le lyrisme français agonisait dans la tiédeur des chapelles : en le laïcisant [...] elles lui restituèrent une vigueur nouvelle [...] En même temps, par la seule audace de leurs confessions, elles mettaient fin à une fastidieuse 
littérature de fausse psychologie ; [...] elles replaçaient la poésie dans la vie, la femme dans la réalité.

\section{Vers l'après Femina, dans les pas de Colette 4.1. Les limites d'une influence}

La revue ne changea guère sa ligne éditoriale au fil des ans. La nouvelle célébration amoureuse choisie par les femmes ne rendit pas Femina plus libre de ton. Les lauréates de son tournoi restent honorées - ainsi des poèmes inédits d'Hélène ornent le numéro spécial, en couleurs, du $1^{\text {er }}$ décembre 1908 et 1909 - l'initiative d'un concours nouveau vise même à élire une Académie féminine idéale (15 avril 1909). Hélène Picard figure encore parmi les élues.

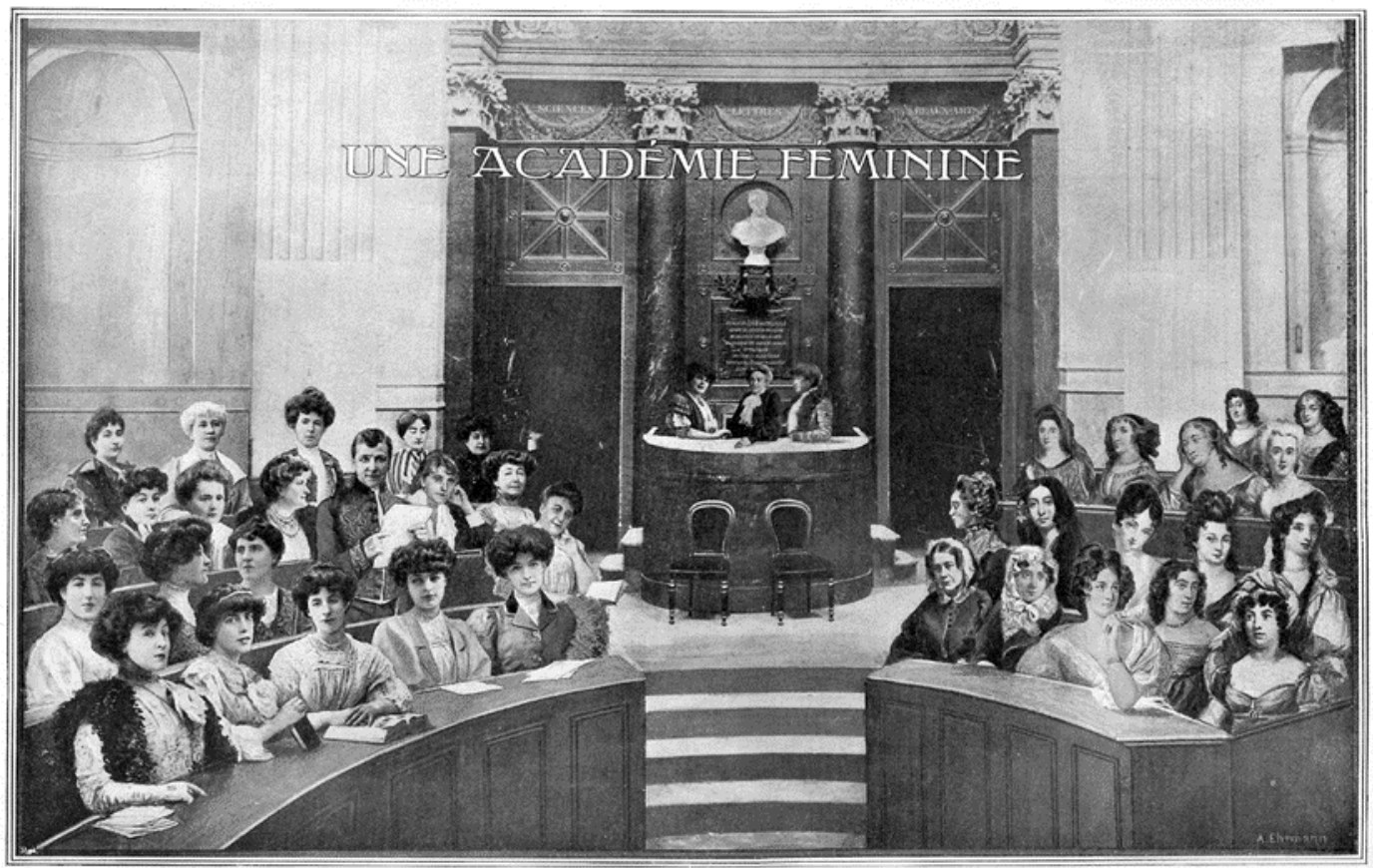

Le montage est à la fois judicieux et grossier : le visage de chacune est greffé à des corps visiblement différents, en tout cas mal proportionnés. Mais les rangs de sièges de part et d'autre du bureau central, lui-même sis entre deux colonnes sous un buste de célébrité, confèrent à l'ensemble l'audace d'une nouveauté en puissance!

La fête de la Poésie, devenue institution, tant elle participait à la notoriété du périodique, s'ouvre en 1910 par un bilan qui semble relever de la performance. Mais il cache aussi sous l'éloge une manière d'autosatisfaction : pouvait-il en être autrement, dans les mots d'une duchesse de Rohan?

[...] je songe à l'impossible tâche que semblaient avoir assumée ceux qui, pour la première fois, il y a dix ans, rêvèrent d'organiser des joutes poétiques féminines [...]. Qui répondrait à l'appel ? Quel serait le poids des gerbes 
moissonnées ? Y avait-il en France tant de talents féminins inconnus?

Hélène Picard ne cessera plus d'écrire, mais hors de l'étroite sphère de Femina. Elle n'a d'ailleurs plus droit qu'à un entrefilet de Henri Duvernois le 15 novembre 1911, l'année où les éditions Sansot publient son premier volet de Souvenirs d'enfance. Le tome II restera sans écho deux ans plus tard, comme l'avait été Fresques auparavant.

\subsection{Des chemins nouveaux}

Ce recueil, paru un an à peine après L'Instant éternel, lui valut pourtant l'intérêt d'une revue italienne à vocation internationale, Poesia. Fondée en 1905 par le père du futurisme, Filippo Tommaso Marinetti, elle publiait des textes français de poètes et poétesses telles Lucie Delarue-Mardrus, Gérard d'Houville / Marie de Régnier, Renée Vivien. Quatre inédits d'Hélène passent ainsi la frontière à trois reprises $\left(\mathrm{n}^{\circ} 8\right.$ de septembre $1908, \mathrm{n}^{\circ} 10$ de novembre, $\mathrm{n}^{\circ}$ 11-12 pour décembre 1908 -janvier 1909). Marinetti prônera dans son manifeste de 1909 un « art de vie explosive » nourri de l'élan vital de Bergson et des paroxysmes de D’Annunzio. On peut se demander comment la poésie d'Hélène put être intégrée à ce mouvement radical, qui se voulait révolutionnaire. Le violent lyrisme de Fresques comme de L'Instant éternel suffit sans doute au critique Paolo Buzzi, (n ${ }^{\text {os }}$ 3-4-5-6 d'avril-juillet 1909), saluant une voix «tutte soffuse di liberazione e di modernità » ( « tout imprégnée de libération et de modernité »!).

Quand le premier conflit mondial appela les femmes à un effort de guerre sans précédent, leur abondante contribution poétique n'en fut pas exclue. Femina n'ayant pas vocation à un engagement de cette nature, Hélène Picard se tourna vers Les Annales politiques et littéraires de façon régulière. Dix-huit poèmes jalonnent ainsi la revue entre août 1915 et l'hiver 1918, tous oubliables par leurs accents cocardiers. L'édition d'un recueil de guerre, Rameaux, chez Fayard en 1919, marqua la fin d'une époque et la fin d'une vie provinciale : Hélène Picard quitte alors son époux et part vivre à Paris. Elle s'y installait lorsque Femina, revenue à son esprit d'antan, accueillit ses deux ultimes contributions à la revue qui l'avait lancée, avec

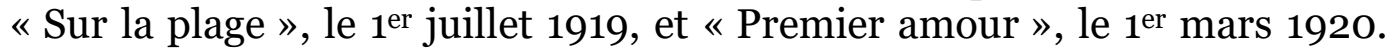
Aussi sages que par le passé, elles ne reflétaient plus l'ambition littéraire d'une femme libérée.

\subsection{Les bouleversements d'une écriture}

Ses premiers pas la portèrent vers les bureaux du Matin, où elle souhaitait rencontrer Colette et lui offrir Les Lauriers sont coupés, deuxième tome de ses Souvenirs d'enfance, paru en 1913. Dédié à la native de la Puisaye dont elle connaît les œuvres et la sensibilité au vivant, le recueil fut son meilleur laisser-passer : les deux provinciales, chacune riche d'un accent 
marqué, s'apprécièrent instantanément. Colette en fit sa secrétaire à la rubrique « Contes des mille et un matins » et lui garda jusqu'à sa mort (1945) une tutélaire amitié.

La ferveur poétique de la Belle Époque avait expiré avec l'avènement surréaliste des années 20, mais rien n'empêchait les poétesses de saisir l'air du temps et ses opportunités. Hélène Picard ne bénéficiait plus de son honorable notoriété, mais de nature audacieuse, curieuse de tout, dotée d'une intuition aiguë qui bluffait Colette elle-même, elle sut percevoir la puissance créatrice des nouveaux courants artistiques, comprenant qu'il fallait oublier ses romantiques idoles.

Une passion secrète pour Francis Carco, ami de Colette, acheva sa propre révolution. Au rude contact du poète qui hantait la pègre parisienne, attirée par le Paris du music-hall, des marges et ses quartiers populaires, elle mit ce monde en scène dans un ouvrage surprenant Pour un mauvais garçon. Publié en 1927 après six ans de gestation, soutenu par Colette qui ne manquait pas d'entregent, il n'eut pourtant pas le temps de briller, l'éditeur André Delpeuch traversant alors de lourds embarras financiers. Mais la critique, même minime, sentit la singularité créative du recueil. Le journal Volonté du 24 juin 1927 souligne un lyrisme indissociable d'une " volupté de la déchéance ». Le 29 octobre de la même année, Paul Fierens des Nouvelles littéraires se dit « poursuivi par des couleurs comme en liberté » par une " voix [c'est lui qui souligne] qui s'encanaille mais qui est prenante, chaude ». André Fontainas au Mercure de France (livraison du 15 septembre 1927) caractérise au plus juste l'esprit de l'œuvre :

$\mathrm{M}^{\mathrm{me}}$ Hélène Picard, le cœur ému d'orgueil à la pensée qu'un Verlaine a vécu dans ces parages où l'infâmie est innocente, situe parmi les bouges d'hôtels borgnes, et de sordides marchands de vins, parmi les carrefours et les ruelles hantées de fille, le roman de son « mauvais garçon».

Nous sommes aux confins de L'Instant éternel ! Peu à peu a éclos un objet rare, unique : l'alexandrin y est heurté, rompu, malmené par de constants rejets ; le langage vert se fond à l'argot de la Butte, peuplé de brownings, couteaux, meublés sordides et vapeurs d'absinthe. Une fille de joie arpente la nuit en quête de son mauvais garçon, entre néons, cris de la rue, musique de cirque et bars louches. Les ruptures cubistes, les tons fauves de Van Dongen ou Vlaminck, les spirales selon Delaunay, les aubes grises d'Utrillo, tout un spectacle urbain surgit, saturé d'échos de jazz ou de beuglants :

Cinés. Autos. Phonos. Clowns... et même, un malheur :

Une femme qu'un geste brusque congédie.

Mais toi ! Avec quel chic tu suis cette douleur

Qu'une enseigne, enfer bleu, incendie.

Un ivrogne est là, qui rit, tout bleu,

Aux reflets des siphons, des carreaux pleins de pluie. [...] 
Les tramways nouent, dénouent leurs rubans de lumière.

Et la poétesse mêle ce monde magnifique et déchu à sa renaissance canaille :

[...] Adieu ! pas de grands mots. Tu les détestes.

Tu m'as appris à remplacer les vieux festons

Par l'affiche imprévue et les couleurs célestes. [...]

Poète, t'ai-je dit, prends ton accordéon!

Enfin ! Plus d'éloquence importune et vorace :

Mon romantisme est mort de ton coup de sifflet.

Autant de traits d'autodérision qui signaient lucidement un étrange parcours - un testament littéraire ? Il ne fut pas déshonorant. Elle obtiendra même grâce à lui le prix Renaissance, le 5 mai 1928, décerné chaque année par l'hebdomadaire La Renaissance politique, littéraire et artistique. Il est vrai que son amie Colette, présidant ce prix à l'époque, œuvra bien en faveur de la poétesse désargentée.

\section{Sur les vestiges de Femina...}

L'amnésie institutionnelle, on le sait, marqua longtemps la période cruciale de la Belle Époque littéraire et sa foison de créatrices. Les revues qui les mettaient à l'honneur ont sombré selon la naturelle loi de l'usure et du marché. Mais avant que les études d'aujourd'hui, à vocation universitaire et/ou féministe, n'exhument ces talents perdus, des relais permirent leur possible survie : le prix Femina, toujours en vigueur de nos jours, est issu d'une précédente distinction que décerna le mensuel féminin Vie heureuse dès la première décennie du $\mathrm{XX}^{\mathrm{e}}$ siècle. Mais le fameux tournoi initié en 1903 participa, à sa mesure certes, à un essor féminin louable en matière de poésie, jusqu'en 1914 .

De son côté, Colette prenait ses marques de romancière dans les années 20, pouvant ainsi contribuer à préserver Hélène Picard d'un naufrage absolu. Si L'Instant éternel porte le sceau maintenant peu lisible des poèmes « Belle Époque », le recueil de 1927, amorcé dès sa nouvelle vie parisienne, mérite un regard passablement admiratif. Hélène elle-même, bien que son image littéraire ne la tourmentât guère, s'en ouvrit à son amie dans une lettre, le 8 mai 1928 :

André Billy, dans l'histoire littéraire qu'il publie me fait, paraît-il, un sort d'outre-tombe extraordinaire et annonce ma célébrité éternelle. [...] Je voudrais simplement un peu plus de justice et de courtoisie de mon vivant. [...] Mais j'espère quelque revanche un jour : simplement le bénéfice de l'équité (Colette, 1988 : 98).

On notera le « paraît-il » qu'insère l'épistolière. En effet nous n'avons pas trouvé trace dans sa Littérature française contemporaine d'une mention de l'ouvrage. Il s'agit plutôt d'une critique parue dans L'Euvre où Billy 
était alors critique littéraire. Voilà toutefois sous la plume d'Hélène une formule émouvante et légitime, applicable à bien des destins de poétesses. En cela aussi, le cas d'Hélène Picard reste exemplaire.

\section{RÉFÉRENCES BIBLIOGRAPHIQUES}

BERTAUT, Jules (1909) : Les Muses françaises d'aujourd'hui. Paris, Librairie des Annales.

BILLY, André (1927) : La Littérature française contemporaine. Paris, Colin.

BonNEFON, Jean de (1909) : La Corbeille des roses ou les dames de lettres. Paris, éditions de Bouville et $\mathrm{C}^{\mathrm{ie}}$.

BuRnAT-PROvins, Marguerite (2006 [1907]) : Le Livre pour toi. Paris, éditions de la Différence.

COLETTE (1988 [1958]) : Lettres à Hélène Picard. Paris, Flammarion.

DAUGUET, Marie (2016 [1904]) : Par l'amour. Paris, Hachette Livre Bnf.

DELMAS, Clément (1987) : La Femme et son destin au miroir de la poésie féminine (1899-1920). Thèse d'État sous la direction de Michel Décaudin, Université Paris III.

DoRNIS, Jean (1912) : La Sensibilité dans la poésie française contemporaine (1885-1912). Paris, Arthème Fayard.

Flat, Paul (1909) : Nos femmes de lettres. Paris, Librairie académique Perrin.

Gourmont, Jean de (1910): Muses d'aujourd'hui. Paris, Mercure de France.

LAVAL-TURPIN, Nicole (2000) : Une femme-poète de la Belle Époque à l'entredeux-guerres. Thèse d'État sous la direction de Julie Bertrand-Sabiani, Université d'Orléans

LAVAL-TURPIN, Nicole (2002) : « Hélène Picard, dans l'ombre bleue de Colette ». Cahiers Colette, 24 (Ateliers), 107-122.

LAVAL-TURPIN, Nicole (2003) : « Hélène Picard et le tournoi des poétesses ». Histoires littéraires,15, 64-79.

LEROY, Géraldi \& Julie SABIANI (1999) : La Vie littéraire à la Belle Époque. Paris, P.U.F.

NOAILLES, Anna de (1901) : Le Coeur innombrable. Paris, Calmann-Lévy.

PERDRIEL-VAISSIÈRE, Hélène (1907) : Celles qui attendent. Paris, Sansot.

PICARD, Hélène (1907) : L'Instant éternel. Paris, Sansot.

PICARD, Hélène (1908) : Fresques. Paris, Sansot.

PICARD, Hélène (1911) : Souvenirs d'Enfance I, Nous n’ïrons plus au bois. Paris, Sansot.

PICARD, Hélène (1013) : Souvenirs d'Enfance II, Les Lauriers sont coupés. Paris, Sansot.

PICARD, Hélène (1919) : Rameaux. Paris, Arthème Fayard.

PICARD, Hélène (1927) : Pour un mauvais garçon. Paris, Delpeuch. 
Çédille, revista de estudios franceses, 20 (2021), 53-72

Nicole Laval-Turpin

SÉCHÉ, Alphonse (1908-1909) : Les Muses françaises. Paris, Michaud. 